\title{
All-optical spin switching under different spin configurations
}

\author{
G. P. Zhang* and Mitsuko Murakami \\ Department of Physics, Indiana State University, Terre Haute, IN 47809, USA
}

(Dated: February 11, 2020)

\begin{abstract}
All-optical spin switching represents a new frontier in femtomagnetism. However, its underlying principles are quite different from traditional thermal activated spin switching. Here, we employ an atomic spin model and present a systematic investigation from a single spin to a large system of over a million spins. We find that for a single spin without an external perturbation, the conservation of total angular momentum requires that the spin change, if any, exactly matches the orbital momentum change, but a laser pulse significantly alters this relation, where the spin change does not necessarily follow the orbital change. This is reflected in the strong dependence of switching on laser polarization. To have an efficient spin switching, the electron initial momentum direction must closely follow the spin's orientation, so the orbital angular momentum is transverse to the spin and consequently the spin-orbit torque lies in the same direction as the spin. The module of the spin-orbit torque is $\lambda|\mathbf{S}||\mathbf{r}||\mathbf{P}| \sqrt{\cos ^{2} \alpha+\cos ^{2} \beta-2 \cos \alpha \cos \beta \cos \gamma}$, where $\alpha(\beta)$ is the angle between spin $\mathbf{S}$ and position $\mathbf{r}($ momentum $\mathbf{P})$ and $\gamma$ is the angle between $\mathbf{r}$ and $\mathbf{P}$. These findings are manifested in a much larger system. We find that the spin response depends on underlying spin structures. A linearly polarized laser pulse creates a dip in a uniform inplane-magnetized thin film, but has little effects on Néel and Bloch walls. Both right- and left- circularly polarized light $\left(\sigma^{+}\right.$ and $\sigma^{-}$) have stronger but different effects in both uniform spin domains and Néel walls. While $\sigma^{+}$light creates a basin of spins pointing down, $\sigma^{-}$light creates a mound of spins pointing up. In the vicinity of the structure spins are reversed, similar to the experimental observation. $\sigma^{+}$light has a dramatic effect, disrupting spins in Bloch walls. By contrast, $\sigma^{-}$light has a small effect on Bloch walls because $\sigma^{-}$only switches down spins up and once the spins already point up, there is no major effect. These findings are expected to have important implications in the future.
\end{abstract}




\section{INTRODUCTION}

Manipulating electron spins in magnetic media with light is interesting, because it underlies the technology behind magneto-optical recording in rare-earth and transition-metal alloys [1]. After a laser beam heats a sample over its compensation point, a magnetic field is applied to switch the spin to a different direction [2, 3]. It is also known that if the sample has a compensation temperature slightly higher than ambient temperature, the demagnetizing field plays a role as a bias field for thermomagnetic writing, free of a magnetic field [4]. This is an earlier version of all-optical spin switching (AOS), but it did not touch the time scale involved. In 2007, the same material showed AOS but on a much faster time scale [5]. To this end, a large number of materials have been found to show AOS [6], besides rare-earth transition metal alloys [7]. Many synthesized alloys show the same behavior, and recently even a Pt/Co/Pt ferromagnetic stack showed AOS [8]. Parlak et al. [9] further demonstrated AOS in CoPt multilayers, which can be further optimized [10].

However, the microscopic mechanism of AOS is still under debate (see reviews [11, 12]). The proposed mechanisms generally fall into two categories: Helicity-dependent (HD) and helicity-independent (HID) switching. Helicity refers to how the electric field of light rotates with respect to the light propagation direction. Figure 1 shows an example of left-circularly polarized light $\left(\sigma^{-}\right)$. It was originally thought that the compensation temperature is critical to ultrafast AOS just as that in slow AOS, but this is not always the case. Hassdenteufel et al. [13] and Schubert et al. [14] showed that switching can appear below or above the magnetic compensation point or in a sample without a compensation point. These two types of switching can appear in the same material, depending on the laser field fluence [15]. Experimentally when a laser pulse scans across their magnetic sample, at the center of laser excitation the domain is demagnetized, with randomly distributed spin up and down domains, and the spin switching appears in the vicinity of the demagnetized region [5]. We recently showed that when one has a ferrimagnet [16], care must be taken because two different helicities act upon two respective sets of spins, leading to apparent HID switching. Because optical selection rules depend on the spatial orientation of spins in a domain "16 18], even subject to the same laser beam, spins inside the domain wall behave differently. This was observed experimentally in multiferroic $\mathrm{TbMnO}_{3}$ [19] which shows a significant dependence on spin configuration. Manz et al. [19] demonstrated a chirality reversal of 
multiferroics by a linearly polarized laser pulse which induces a skymion structure through magnetoelectric interactions.

In this paper, we aim to carry out a systematic investigation as how system parameters and laser parameters affect all-optical spin switching. We employ a model that takes into account the spin-orbit coupling, spin-spin interaction and the interaction between the laser field and system, without resorting to an effective magnetic field [15]. We start with a single spin to demonstrate that a laser pulse can significantly decouple the spin change from the orbital angular momentum change. We find that rather than through momentum exchange with the orbital, the spin change is better described by the spin-orbit torque. This is revealed by using different laser polarizations and initial spin orientations. By changing the direction of momentum, we find that to reverse spins, it is necessary to have a momentum along the spin direction, so the transverse momentum is boosted to generate a large longitudinal torque on the spin. These intricate relations are manifested in a thin film of a million spins. We employ three different types of magnetic domains: a uniform inplane magnetized domain, Néel and Bloch walls. The same linearly polarized light induces very different changes. The uniform magnetized film shows a dip at the center of excitation, but Néel and Bloch walls show a much weaker change. Circularly polarized light has a much stronger effect. We find that circularly polarized light can directly write information into a uniform spin domain, Néel and Bloch walls. A circular pattern is formed and around the vicinity of the pattern, the spins are reversed, similar to the experimental results [5]. At the center of excitation, spins are disoriented and chaotic, also similar to the experiment [5]. Right- $\left(\sigma^{+}\right)$and left$\left(\sigma^{-}\right)$circularly polarized light have different effects. $\sigma^{+}$tends to flip spins from up to down, while $\sigma^{-}$does the opposite. This explains why $\sigma^{-}$does not change the Bloch wall strongly. Our findings shed fresh light on AOS and are expected to have important impacts on future experimental and theoretical investigations.

The rest of the paper is arranged as follows. In Sec. II, we outline our theoretical formalism. Section III is devoted to the results and discussions. We present our findings on a single spin first and then on domains of three different types, uniform domains with inplane magnetization in Sec. III(C), Néel walls in Sec. III(D) and Bloch walls in Sec. III(E). We provide an analytic theory on spin-orbit torque in Sec. III(F). Finally, we conclude this paper in Sec. IV. An appendix is provided to discuss the role of orbital angular momentum. 


\section{THEORETICAL FORMALISM}

All-optical spin switching employs an ultrafast laser pulse to switch spins in a magnetic material. Figure 1(a) represents a typical experimental geometry with lattice sites $\left(N_{x} \times\right.$ $N_{y} \times N_{z}$ ), where the pulse is propagating along the $-z$ axis and illuminates a small area of the sample with radius $R$. The pulse can be either linear or circularly polarized. The magnetic films contain many spins, with various domain structures. Any reasonable theory must treat both the laser field and system properly. AOS is an optical process. Under dipole approximation, light does not interact with spin because the selection rule dictates spin conservation, or $\Delta S=0$. This naturally creates a major problem for theoretical treatment. To circumvent this difficulty, one often introduces a spin-symmetry breaking term such as an effective magnetic field [15] to simulate laser pulses. This effective field should not be confused with the laser's own magnetic field. First-principles calculations are possible, but are unable of reproducing spin reversal [16, 20].

When we were investigating the magneto-optical effect, we accidentally discovered a model [21, 22] that allows us to simulate spin reversal, without an effective magnetic field. Different from other studies, we augment a spin-orbit coupling term, $\lambda \mathbf{L}_{i} \cdot \mathbf{S}_{i}$, to the Heisenberg exchange model, where $\lambda$ is the spin-orbit coupling constant, $\mathbf{L}_{i}$ and $\mathbf{S}_{i}$ are the orbital and spin angular momenta at site $i$. Our Hamiltonian is [17, 21 23]

$$
H=\sum_{i}\left(\frac{\mathbf{P}_{i}^{2}}{2 m}+V\left(\mathbf{r}_{i}\right)+\lambda \mathbf{L}_{i} \cdot \mathbf{S}_{i}-e \mathbf{E}(t) \cdot \mathbf{r}_{i}\right)-J \sum_{i j} \mathbf{S}_{i} \cdot \mathbf{S}_{j}
$$

where the first term is the kinetic energy operator of the electron, the second term is the potential energy operator, and the third term is the spin-orbit coupling. The fourth term in Eq. 1 describes the interaction between the laser pulse $\mathbf{E}(t)$ and the system [21], so we do not have to employ an effective magnetic field approximation. The laser field, centered with respect to magnetic domains, propagates vertically down along the $-z$ axis, with the electric field

$$
\mathbf{E}(\mathbf{r}, t)=\mathbf{A}(t) \exp \left[-\frac{\left(x-x_{c}\right)^{2}+\left(y-y_{c}\right)^{2}}{R^{2}}-\frac{z}{d}\right]
$$

where $x$ and $y$ are the coordinates in the unit of the site number, $x_{c}$ and $y_{c}$ denote the center location of the laser spot, $R$ is the radius of the laser spot ( $R$ is 50 lattice sites in our simulation, see Fig. 1(a)), and $d$ is the penetration depth of light ( $d=30$ lattice sites in our 
case). For a left (right)-circularly polarized field $\left[\sigma^{-}\left(\sigma^{+}\right)\right], \mathbf{A}(t)$ is

$$
\mathbf{A}(t)=A_{0} \mathrm{e}^{-t^{2} / T^{2}}[\mp \sin (\omega t) \hat{x}+\cos (\omega t) \hat{y}]
$$

where $\omega$ is the laser carrier frequency, $T$ is the laser pulse duration, $A_{0}$ is the laser field amplitude, $t$ is time, and $\hat{x}$ and $\hat{y}$ are unit vectors, respectively. For a linearly polarized field $(\pi), \mathbf{A}(t)$ is

$$
\mathbf{A}(t)=A_{0} \mathrm{e}^{-t^{2} / T^{2}} \cos (\omega t) \hat{x}
$$

These three fields are used in the following calculation. The laser pulse duration $T$ is 60 fs and the field amplitude takes several different values (see below for details). Figure 1(a) shows our simulation box with $N_{x}=501$ spins along the $x$ axis, $N_{y}=501$ spins along the $y$ axis, and $N_{z}=4$ spins along the $z$ axis. We take into account three types of spin configuration: (i) uniform spin domains, (ii) Bloch and (iii) Néel walls. The domain wall width for both Néel and Bloch walls is 200 lattice sites. The last term in Eq. (1) is the exchange coupling between two neighboring spins, and $J$ is the exchange parameter. We choose $S=1 \hbar, J=0.1 \mathrm{eV} / \hbar^{2}, \lambda=0.06 \mathrm{eV} / \hbar^{2}[24]$. We adopt the harmonic oscillator potential for $V(\mathbf{r})$. This Hamiltonian is similar to the traditional harmonic oscillator model for electron in magneto-optics [21].

To compute spin dynamics, we solve the equation of motion for each operator $O$ of interest, $i \hbar \dot{O}=[O, H]$. For instance, the spin changes according to

$$
\frac{d \mathbf{S}_{i}}{d t}=\lambda \mathbf{L}_{i} \times \mathbf{S}_{i}+J \sum_{j} \mathbf{S}_{i} \times \mathbf{S}_{j}
$$

Since our main interest is to compute a system with many spins, we make a Hartree-Fock approximation to the coupled operator, where operators are replaced by their expectation values. More accurate calculations are possible, but then one is restricted to a system size too small to compare with experimental sizes. This is the compromise that we have to make.

\section{RESULTS AND DISCUSSIONS}

To develop a physical picture for AOS, we start with a single spin and then move on to more complex systems with a million exchange-coupled spins. The results on a single spin serve the basis for our research. 


\section{A. Single spin}

For a single spin, no exchange interaction is present and only the spin-orbit coupling remains. Under influence of spin-orbit coupling, the spin at site $i$ precesses according to

$$
\frac{d \mathbf{S}_{i}}{d t}=\lambda \mathbf{L}_{i} \times \mathbf{S}_{i}
$$

The right hand side is the spin-orbit torque, and depends on the orbital and spin itself. The orbital angular momentum precesses as

$$
\frac{d \mathbf{L}_{i}}{d t}=-\lambda \mathbf{L}_{i} \times \mathbf{S}_{i}
$$

So one can see that for a single spin system, $\mathbf{S}_{i}+\mathbf{L}_{i}$ at each lattice site $i$ is conserved, recovering the familiar conservation law of total angular momentum. This means that the amount of spin change must be matched exactly by the orbital angular momentum change. What is less familiar, however, is the orbital angular momentum change due to a laser field. With presence of a laser beam, Eq. (17) becomes

$$
\frac{d \mathbf{L}_{i}}{d t}=-\lambda \mathbf{L}_{i} \times \mathbf{S}_{i}-e \mathbf{E}(t) \times \mathbf{r}_{i}(t)
$$

where $\mathbf{E}(t)$ is the laser electric field and $\mathbf{r}_{i}(t)$ is the position which also depends on the laser field. This extra term is from the laser field and is the main course of the total angular momentum J change (see Fig. 2(a)). However, the spin does not have this extra term, so Eq. (6) remains valid even with presence of laser. If we add Eqs. (66) and (8) and move the orbital term to the right side, we find

$$
\frac{d \mathbf{S}_{i}}{d t}=-\frac{d \mathbf{L}_{i}}{d t}-e \mathbf{E}(t) \times \mathbf{r}_{i}(t)
$$

Integrating Eq. (9), we find

$$
\mathbf{S}_{i}(t)-\mathbf{S}_{i}(-\infty)=-\int_{-\infty}^{t} d \mathbf{L}_{i}-e \int_{-\infty}^{t} \mathbf{E}\left(t^{\prime}\right) \times \mathbf{r}_{i}\left(t^{\prime}\right) d t^{\prime}
$$

Since $-e \mathbf{E}(t)$ is the force on the electron and $\mathbf{r}_{i}(t)$ is the time-dependent position, the last term in the above equation is the time-integrated torque. The amount of spin change is directly proportional to the accumulated torque. Therefore, a large spin change is possible for a weak laser pulse as long as its pulse duration is long. This is the essence of our theory. The appendix has a more detailed discussion on orbital angular momentum.

(February 11, 2020) 
Next we show a numerical example to demonstrate many interesting aspects of AOS. We choose a linearly polarized pulse along the $y$ axis, with the field amplitude of $0.09 \mathrm{~V} / \AA$. The field amplitude is chosen to maximize the spin switching. The photon energy is $1.6 \mathrm{eV}$ and the pulse duration is $60 \mathrm{fs}$. Initially, the spin points along the $-z$ axis. The initial momentum of the electron is $9.11 \times 10^{-26} \mathrm{kgm} / \mathrm{s}$, which corresponds to a velocity of $1 \AA /$ fs, close to the Fermi velocity in metals, along the $z$ axis. Figure 2 shows three components of the total angular momentum J, spin and orbital angular momentum as a function of time. Our laser pulse peaks at 0 fs. From Fig. 2(a), one can see that before and after laser pulse excitation the total angular momentum $\mathbf{J}$ is conserved. Figure 2(a) demonstrates clearly that the $z$ component $J_{z}$ starts out with $-1 \hbar$ and ends around $+0.75 \hbar$, which occurs during interaction with the laser field. Different from $J_{z}, J_{x}$ is zero in the beginning, and as the laser field excites the system, it becomes negative. For our current spin and momentum configuration, $J_{y}$ is zero. To understand these features, we further plot the spin and orbital angular momenta separately. Figure 2(b) shows that the orbital angular momentum $L_{x}$ undergoes a rapid oscillation around zero after laser excitation. This is due to the laser-induced torque (the last term in Eq. 8). Spin dynamics is different. We notice that $S_{x}$ undergoes a vertical shift to the $-x$ axis and then oscillates. After excitation, the spin and orbital momentum oscillate exactly out of phase because their spin-orbit torques differ by a negative sign. This leads to the constant $J_{x}$ seen in Fig. 2(a). Figure 2(c) reveals the reason behind $J_{y}=0$, where $L_{y}$ and $S_{y}$ always oscillate out of phase with each other. We emphasize that this is the direct consequence of our current spin and momentum configuration. Whether the spin oscillates or not depends on both the laser parameters and the spin value (see another example below).

The $z$ components of the spin and orbital angular momentum are shown in Fig. 2(d). We see that $S_{z}$ switches from $-1 \hbar$ to $0.75 \hbar$, but $L_{z}$ only oscillates around zero. This is consistent with the finding along the $x$ axis. The laser field directly injects angular momentum into the orbital degree of freedom, wherein both the $x$ and $z$ components undergo a rapid oscillation around 0 fs because the position and momentum are directly influenced by the laser field. Equation (8) shows that the orbital angular momentum change is subject to two separate contributions. Since the laser frequency is much higher than that of SOC, the rapid beating seen in the orbital angular momentum reflects that the laser field dominates the process over the spin-orbit coupling. Equation (9) shows that the rapid component in $d \mathbf{L} / d t$, which 
is a torque itself, compensates the torque due to the laser field. This compensation is the manifestation of the dipole selection rule that the photon angular momentum is absorbed by the electron's orbital angular momentum. In other words, the rapid oscillation is consumed by the orbital angular momentum as can be seen from Eq. (8). The spin change is still from Eq. (6). Because the right-hand side is a cross-product, a $z$ component of the spin $S_{z}$ depends on the $x$ and $y$ components of the spin and orbital angular momentum $\left(\tau_{z}=\lambda\left(L_{x} S_{y}-L_{y} S_{x}\right)\right)$. Since we align the spin along the $z$ axis, both $S_{x}$ and $S_{y}$ are zero in the beginning. Therefore, to change $S_{z}$, one has to wait until $S_{x}$ and $S_{y}$ differ from zero, and the direct and rapid response seen in the orbital angular momentum does not occur to spins. Spin has SU(2) symmetry and must interact with the laser field even times to be affected [25]. This is an important character of AOS. We will come back to this below.

\section{B. Effects of spin, momentum and laser-field on spin switching}

In general, not all the spins in domain walls point in the same direction in space. Even illuminated by the same type of light, these spins respond differently since their local quantization axes are different. A z-component for one spin could become an $x$ component for another. Therefore, without considering spin configuration explicitly, it is very difficult to develop a sound physical intuition of spin reversal. Our study examines how spin switching depends on the laser field direction. Following the above discussion, we investigate a case where both the initial spin $\mathbf{S}_{0}$ and initial momentum $\mathbf{P}_{0}$ point along the $-z$ axis. The direction of the laser field $\mathbf{E}$ is very critical to AOS. For instance, if we align the laser electric

field and the initial spin along the same direction, i.e., $\mathbf{E}\left\|\mathbf{S}_{0}\right\| \mathbf{P}_{0}$, then the orbital angular momentum is going to be zero. From Eq. ([6), we see the torque is zero, so the spin can not be reversed.

We next consider the laser field $\mathbf{E}$ along the $y$ axis, perpendicular to $\mathbf{S}_{0}$ and $\mathbf{P}_{0}$. Figure 3(a) shows that three components of spin behave differently. $S_{x}$ proceeds to a large negative value and oscillates, but $S_{y}$ shifts a little and immediately oscillates after laser peaks. $S_{z}$ shows a much stronger change and flips from the $-z$ axis to the $+z$ axis. Now if we rotate $\mathbf{E}$ to the $x$ axis with respect to the $z$ axis by $-90^{\circ}$, the spin also rotates by $-90^{\circ}$. If we 
compare Figs. 3(a) and 3(b), we can figure out the transformation matrix as

$$
Q_{1}=\left(\begin{array}{rrr}
0 & -1 & 0 \\
1 & 0 & 0 \\
0 & 0 & 1
\end{array}\right)
$$

Next we keep $\mathbf{E}$ along the $y$ axis but flip the spin from the $-z$ to $+z$ axis. Figure 3 (c) shows that $\mathbf{S}$ undergoes a $180^{\circ}$ rotation along the $x$ axis, with a different rotation matrix

$$
Q_{2}=\left(\begin{array}{rrr}
1 & 0 & 0 \\
0 & -1 & 0 \\
0 & 0 & -1
\end{array}\right)
$$

One sees that the spatial orientation of spin determines how light affects the spin. If we keep the spin along the $+z$ axis but rotate $\mathbf{E}$ to the $x$ axis as we did in Fig. 3.(b), we find the same transformation matrix $Q_{1}$ (Fig. 3(d)). To comprehensively understand how the initial momentum $\mathbf{P}_{0}$ affects spin dynamics, we fix its magnitude, but change its spatial orientation through $(\theta, \phi)$, whose definitions are presented in Fig. 1(b). We choose two different spin angular momenta $S_{0}=1 \hbar$ and $2 \hbar$ since it is known the spin value affects how laser switches spins [22]. Figure 4(a) plots the final spins for $S_{0}=+1 \hbar$ as a function of $\theta$ for $\phi=0^{\circ}$ and $90^{\circ}$. Figure 4 (a) makes abundantly clear that spatial orientation of $\mathbf{P}_{0}$ has a significant effect on spin reversal. The spin reverses only if $\theta$ is close to $0^{\circ}$ or $180^{\circ}$ (see the large and negative final spins in Fig. 4(a)). $\phi$ has a small effect (compare the results for $\phi=0^{\circ}$ and $180^{\circ}$ ), meaning that the transverse momentum has no major effect. Consistent with prior results [22], the spin itself also affects this dependence. In Fig. 4(b), we use a larger spin of $2 \hbar$, and we find that the spin switchability is better, without a small oscillation seen in Fig. 4(a), but the general conclusion remains the same.

\section{Switching uniform spins}

In real magnetic materials, domains come with different shapes and spin structures. Different from the above single spin studies, spins are exchange coupled together. In the following, we investigate how these different spin structures affect the spin reversal process. We start with uniform spins initialized along the $y$ axis (see Fig. 5(a)) with the same magnitude of $1 \hbar$. The initial momentum $\mathbf{P}_{0}$ is also in the $y$ direction, so we have an optimal 
condition. Following our finding in the single spin, we apply a linearly polarized pulse along the $x$ axis, which allows a nonzero orbital angular momentum. Spins precess in the time domain. Figure 5(b) shows a snapshot at $123 \mathrm{fs}$, after the laser pulse peaks. This particular time is selected because it is after laser excitation and its spin change is representative. The color bar denotes $S_{z}$ at each lattice site. One sees that spin activities concentrate around the center of the excitation where the laser field is strongest. Spins clearly tilt toward the $-z$ axis and form a dip. Around the circumference of the dip, spins smoothly transition into the background. Note that to reduce the huge file size, we only plot one out of every ten spins. When we switch to a right-circularly polarized pulse $\left(\sigma^{+}\right)$, we note a dramatic change. Figure 5(c) reveals that the spin change becomes larger (compare Figs. 5(b) and 5(c)). For instance, we notice that spins in the active region point down and form a basin, and in the center some spins are along the $x$ axis as well as the $-y$ axis, i.e., inplane spin reversal, though the majority of spins point toward the $-z$ axis. The situation is reversed if we use left-circularly polarized light $\left(\sigma^{-}\right)$. Figure $5(\mathrm{~d})$ shows that spins point along the $+z$ axis and form a mound. This is consistent with our theory [16] that $\sigma^{+}$flips upspin down, while $\sigma^{-}$flips downspin up.

\section{Switching spins in a Néel wall}

A good place to understand the effect of the initial spin configuration on AOS is to investigate what happens if the laser pulse impinges on a Néel wall. Here, spins within the wall take different spatial orientations, so locally each site has a different quantization axis. By studying their switchability, we can learn the crucial connection between the spin and AOS. The wall is created by two functions, $S_{i}^{x}=S_{0} \cos \left(\xi_{i}\right)$ and $S_{i}^{y}=S_{0} \sin \left(\xi_{i}\right)$, where $S_{0}=1 \hbar$, and $i$ is the lattice site index running from $W_{L}$ to $W_{R}$. $\xi_{i}=i \pi /\left(W_{L}-W_{R}\right)-$ $\pi\left(W_{L}+W_{R}\right) /\left(W_{L}-W_{R}\right) / 2$, where $W_{L}$ and $W_{R}$ are the left and right limits of the wall, respectively. $W_{L}$ and $W_{R}$ determine the width of the wall. In our case, the width is 200 sites. This creates a Néel wall as illustrated in Fig. 6(a). Spins with lattice site indices less than $W_{L}$ point in the $+y$ direction, and spins with lattice site indices larger than $W_{R}$ point in the $-y$ direction. The second term in $\xi_{i}$ ensures that spins in the middle of the wall point along the $+x$ axis.

We first subject the sample to a $\pi$ pulse. Figure 6(b) shows a snapshot at 123 fs. Inter- 
estingly, we find that the overall change is small. There is no major domain breakup. Only a small change is noticed in the center. The effect is even smaller than that in the uniform spin case. This indicates that the Néel wall can tolerate laser excitation more than the uniform spin slab. The reason for this difference is that spin switching depends on the initial spin configuration, its relative orientation with respect to the laser electric field direction and the area of laser beam. The spins inside the Néel wall are parallel to the laser electric field, while the spins in the uniform spin slab are perpendicular to the laser electric field. This difference leads to two different spin-orbit torques acted upon these two spin structures. For the spins outside the Néel wall, their directions are perpendicular to the laser electric field, so they are strongly affected if the laser beam radius is large, just as those in the uniform spin slab. The situation changes when we subject the magnetic slab to a $\sigma^{+}$pulse (Fig. 6(c)), where the spins are switched to the $-z$ axis. What is even more interesting is that the spins around the perimeter of the pattern all point down. This is very similar to the experimental observation where the switched region appears around the circumference [5]. Figure 6(d) plots that $\sigma^{-}$creates a mixed spin domain, where the spins form a pattern tilting toward $+z$ axis and smoothly merging into the original Néel wall.

We also investigate how the laser beam radius affects spin reversal in Néel walls. We apply a linearly polarized pulse along the $x$ axis. Figure 7 shows results for three radii, (a) $R=50$, (b) $R=150$ and (c) $R=200$. The image for $R=50$ (Fig. 7 (a)) is the same as Fig. 6(b), but on a larger scale with $501 \times 501$, so the entire changed area appears to be smaller. Figure 7 (b) shows that as the radius becomes larger, the affected region is larger. However, the spin in the Néel wall is protected, with a very small change. The main change occurs in the vicinity of the excitation center, where the spins are along the $y$ or $-y$ axis and experience a stronger torque. This spatial inhomogeneity persists even if we use $R=200$ which matches the width of the wall (see Fig. 7(c)). Although spins outside the Néel wall switch roughly to the same direction, spins inside the Néel wall do not. This is because spin-orbit torques on those spins inside the Néel wall are different from those outside the Néel wall. Spins inside the Néel wall do not orient in the same direction, and they mainly lie along the $x$ axis, so the domain wall retains. 


\section{E. Switching spins in a Bloch wall}

To this end, the initial spins are in the same plane of the light polarization. In the Bloch wall, spins are along the normal direction. Our domain wall width also has 200 lattice sites. The wall is created by two functions, $S_{i}^{y}=S_{0} \sin \left(\xi_{i}\right)$ and $S_{i}^{z}=S_{0} \cos \left(\xi_{i}\right)$, which smoothly merges into the neighboring domains. The produced spin structure can be seen from Fig. 8(a). We start with a $\pi$ pulse. The results are shown in Fig. 8(b). We see that the effect on the domain wall is very weak. This is similar to the Néel wall (Fig. 66(b)). By contrast, a $\sigma^{+}$pulse has a much stronger effect. Recall that a $\sigma^{+}$pulse switches spin from up to down [16], so the current spin configuration is an ideal place to play a strong role. Here the spin dynamics is violent, with a distinctive feature of spin reversal around the vicinity of the excitation region. In the center spins are much more chaotic. Beyond the excitation center, the domain structure remains intact. We note that the radius of the laser spot is 50 lattice sites. This well localized spin structure of about 200 sites represents a possibility to create a much small magnetic domain, potentially very useful for development of denser magnetic storage devices. We also investigate how $\sigma^{-}$affects the Bloch wall. Figure 8 (d) shows a snapshot at 123 fs that the spins are not strongly affected. This is because $\sigma^{-}$tends to flip spins from down to up, but once spins are already up, the effect on them is very small [16].

We can make a connection to existing experiments. Stanciu et al. [5] showed that spin switching occurs at the vicinity of the demagnetized area. From our study, we indeed observe that the reversed spins mainly appear around the ring of the excitation (see Figs. 6(c) and 6(d) as well as Figs. 8(c) and 8(d)). Note that Stanciu's sample is ferrimagnetic and in principle two different spin sublattices play a role. Our findings serve a theoretical basis for these experimental results and are expected to have important impacts in the future.

\section{F. Spin-orbit torque theory}

The above numerical results present us rich information how spins are affected with different spin configurations. We can reveal further insight into the underlying physics by investigating how the orbital angular momentum enters the picture. We seek the guidance from the spin-orbit torque $\tau=\lambda(\mathbf{L} \times \mathbf{S})$. To be definitive, consider a spin inplane and along the $y$ axis as illustrated in Fig. 9(a), with the laser pulse propagating along the 
$-z$ axis, normal incidence. The laser electric field is decomposed along the $x$ and $y$ axes, respectively. To reverse spin $S_{y}$, one must have a nonzero torque or $\tau_{y} \neq 0$. Because

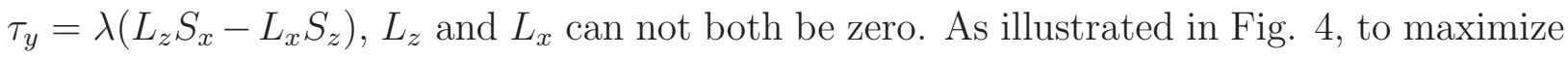
AOS, in our simulation we set the initial momentum along the $y$ axis, same as the spin. For this inplane spin configuration, only the $x$ component $E_{x}$ of the laser field is effective. If the laser polarization is also along the $y$ axis (only $E_{y}$ is nonzero), then the electron only moves along the $y$ axis, generating a zero orbital angular momentum due to $\mathbf{L}=\mathbf{r} \times \mathbf{P}$. Once the orbital angular momentum is zero, the torque is zero, so there is no spin switching. The middle figure of Fig. 9(a) shows that the plane of the orbital angular momentum is perpendicular to the spin, so the torque $\tau$ in the right figure is in the same direction of the spin, so switching is possible.

Figure 9(b) plots the spin pointing out of plane [23]. In this case, the orbital angular momentum plane is in the $x y$ plane (see the middle figure in Fig. 9(b)). Because $\tau_{z}=$ $\lambda\left(L_{x} S_{y}-L_{y} S_{x}\right), L_{x}$ and $L_{y}$ can not both be zero if switching is desired. The inplane orbital angular momentum ensures the resultant torque is along the spin direction (see the right figure in Fig. 9(b)) to switch spins. These two special cases for the spin orientation point out a crucial fact that to switch the spin the orbital angular momentum plane must be perpendicular to the spin.

To see how geometrically the electron position, momentum and spin are intertwined, we rewrite the torque as a triple vector product $\tau=\lambda(\mathbf{r} \times \mathbf{P}) \times \mathbf{S}$,

$$
\tau=\lambda(\mathbf{r} \cdot \mathbf{S}) \mathbf{P}-(\mathbf{P} \cdot \mathbf{S}) \mathbf{r},
$$

which highlights that the torque lies on the plane defined by the momentum and position (see the upper right figure in Fig. 91). If a spin happens to lie in this plane, it will be strongly affected. This explains why in Fig. 4 to switch spin effectively, the angle between the spin and momentum and that between the spin and position must remain small. More importantly we find the module squared of $|\tau|^{2}$ can be written as

$$
|\tau|^{2}=\lambda^{2}|\mathbf{S}|^{2}|\mathbf{r}|^{2}|\mathbf{P}|^{2}\left(\cos ^{2} \alpha+\cos ^{2} \beta-2 \cos \alpha \cos \beta \cos \gamma\right)
$$

where $\alpha$ is the angle between $\mathbf{r}$ and $\mathbf{S}, \beta$ is between $\mathbf{P}$ and $\mathbf{S}$, and $\gamma$ is between $\mathbf{r}$ and $\mathbf{P}$. Here we see that the dependence of the spin-orbit torque on the position and momentum is similar as expected. The smaller the angle is, the larger the torque is. We note that these 
three angles are not independent. For instance, if $\gamma=0^{\circ}$, then $\alpha=\beta$. There is an internal competition between the position and momentum: To have a large torque, the spin has to lie as close as possible to both the position and momentum, but the position and momentum must not align. This competition underlies the theory of spin-orbit torque for all-optical spin switching.

\section{CONCLUSION}

We have shown that laser-induced all-optical spin switching sensitively depends on both the intrinsic properties such as spin structure and initial momentum of electron and the extrinsic laser parameters such as laser polarization. We find that while for a single spin, the total angular momentum is always conserved in the absence of a laser field, a laser pulse fundamentally alters the relation between spin and orbital angular momenta. At least within our current model, the angular momentum transfer between the spin and orbital does not constitute a major channel for spin switching. Instead, the time-integrated spin-orbit torque is the main driver for spin change. Because of this integration, spins can be excited even with a weak laser pulse, provided that the pulse is long enough. We further show that the laser field polarization directly influences the spin vector. Rotating the polarization from the $y$ axis to $x$ axis also rotates the spin vector. However, if we flip the initial spin, the same laser field induces a different spin precession. These findings are manifested in a much larger system. For a magnetic thin film with inplane magnetization, linear $(\pi)$, right and left circularly polarized light $\left(\sigma^{+}\right.$and $\left.\sigma^{-}\right)$induce different changes. $\sigma$ light generates a distinctive pattern of spin reversal, while $\pi$ light only tilts spins. When a light field of the same kind illuminates Néel and Bloch walls, the resultant spin patterns have imprints of the starting domains. Both the Néel and Bloch walls can withstand $\pi$ light, but suffer a dramatic spin change from $\sigma$ light. $\sigma^{+}$and $\sigma^{-}$, both being circular, also have a different effect on the spin. In Bloch walls with the spins pointing along the $+z$ axis, $\sigma^{-}$has little

effect on the wall, but $\sigma^{+}$reverses a major portion of the domain. These findings present a case that can be experimentally tested. Investigation of different spin structures has been a focus recently. Several prior studies have even proposed the domain size [26] as a criterion for the all-optical spin switching. We believe that by examining different spin structures, one can learn more about the interplay between spin, orbital and laser field, which is potentially 
important for future development of fast magnetic storage.

\section{ACKNOWLEDGMENTS}

This work was solely supported by the U.S. Department of Energy under Contract No. DE-FG02-06ER46304. Part of the work was done on Indiana State University's high performance quantum and obsidian clusters. The research used resources of the National Energy Research Scientific Computing Center, which is supported by the Office of Science of the U.S. Department of Energy under Contract No. DE-AC02-05CH11231.

*guo-ping.zhang@outlook.com

[1] M. H. Kryder, Magneto-optic recording technology, J. Appl. Phys. 57, 3913 (1985).

[2] G. P. Zhang, W. Hübner, E. Beaurepaire, and J.-Y. Bigot, Laser-induced ultrafast demagnetization: Femtomagnetism, A new frontier? Topics Appl. Phys. 83, 245 (2002).

[3] A. Kirilyuk, A. V. Kimel, and Th. Rasing, Ultrafast optical manipulation of magnetic order, Rev. Mod. Phys. 82, 2731 (2010). Erratum: Rev. Mod. Phys. 88, 039904 (2016).

[4] H. P. D. Shieh and M. H. Kryder, Magneto-optic recording materials with direct overwrite capability, Appl. Phys. Lett. 49, 473 (1986).

[5] C. D. Stanciu, F. Hansteen, A. V. Kimel, A. Kirilyuk, A. Tsukamoto, A. Itoh, and Th. Rasing, All-optical magnetic recording with circularly polarized light, Phys. Rev. Lett. 99, 047601 (2007).

[6] S. Mangin, M. Gottwald, C-H. Lambert, D. Steil, V. Uhlir, L. Pang, M. Hehn, S. Alebrand, M. Cinchetti, G. Malinowski, Y. Fainman, M. Aeschlimann, and E. E. Fullerton, Engineered materials for all-optical helicity-dependent magnetic switching, Nat. Mater. 13, 286 (2014).

[7] S. Alebrand, M. Gottwald, M. Hehn, D. Steil, M. Cinchetti, D. Lacour, E. E. Fullerton, M. Aeschlimann, and S. Mangin, Light-induced magnetization reversal of high-anisotropy TbCo alloy films, Appl. Phys. Lett. 101, 162408 (2012).

[8] M. Vomir, M. Albrecht, and J.-Y. Bigot, Single shot all optical switching of intrinsic micron size magnetic domains of a Pt/Co/Pt ferromagnetic stack, Appl. Phys. Lett. 111, 242404 (2017). 
[9] U. Parlak, R. Adam, D. E. B̈̈gler, S. Gang, and C. M. Schneider, Optically induced magnetization reversal in $[\mathrm{Co} / \mathrm{Pt}]_{N}$ multilayers: Role of domain wall dynamics, Phys. Rev. B 98, $214443(2018)$.

[10] K T. Yamada, K. Prabhakara, T. Li, F. Ando, S. Semin, T. Ono, A. Kirilyuk, A. V. Kimel, and Theo Rasing, Efficient all-optical helicity-dependent switching in $\mathrm{Pt} / \mathrm{Co} / \mathrm{Pt}$ with dual laser pulses, Arxiv.1903.01941 (2019).

[11] G. P. Zhang, T. Latta, Z. Babyak, Y. H. Bai, and T. F. George, All-optical spin switching: A new frontier in femtomagnetism - A short review and a simple theory, Mod. Phys. Lett. B 30, 1630005 (2016).

[12] G. P. Zhang, M. Murakami, M. S. Si, Y. H. Bai, and T. F. George, Understanding all-optical spin switching: Comparison between experiment and theory, Mod. Phys. Lett. B 32, 1830003 (2018).

[13] A. Hassdenteufel, B. Hebler, C. Schubert, A. Liebig, M. Teich, M. Helm, M. Aeschlimann, M. Albrecht, and R. Bratschitsch, Thermally assisted all-optical helicity dependent magnetic switching in amorphous $\mathrm{Fe}_{100-x} \mathrm{~Tb}_{x}$ alloy films, Adv. Mater. 25, 3122 (2013).

[14] C. Schubert, A. Hassdenteufel, P. Matthes, J. Schmidt, M. Helm, R. Bratschitsch, and M. Albrecht, All-optical helicity dependent magnetic switching in an artificial zero moment magnet, Appl. Phys. Lett. 104, 082406 (2014).

[15] T. A. Ostler, J. Barker, R. F. L. Evans, R. W. Chantrell, U. Atxitia, O. Chubykalo-Fesenko, S. El Moussaoui, L. Le Guyader, E. Mengotti, L. J. Heyderman, F. Nolting, A. Tsukamoto, A. Itoh, D. Afanasiev, B. A. Ivanov, A. M. Kalashnikova, K. Vahaplar, J. Mentink, A. Kirilyuk, Th. Rasing, and A. V. Kimel, Ultrafast heating as a sufficient stimulus for magnetization reversal in a ferrimagnet, Nat. Commun. 3, 666 (2012).

[16] G. P. Zhang, Z. Babyak, Y. Xue, Y. H. Bai, and T. F. George, First-principles and model simulation of all-optical spin reversal, Phys. Rev. B 96, 134407 (2017).

[17] M. Murakami, Z. Babyak, M. Giocolo, and G. P. Zhang, Quantum mechanical interpretation of the ultrafast all-optical spin switching, J. Phys.: Condensed Mat. 29, 184002 (2017).

[18] G. P. Zhang, T. Jenkins, M. Bennett, and Y. H. Bai, Manifestation of intra-atomic 5d6s-4f exchange coupling in photoexcited gadolinium, J. Phys.: Condensed Mat. 29, 495807 (2017).

[19] S. Manz, M. Matsubara1, T. Lottermoser, J. Büchi, A. Iyama, T. Kimura, D. Meier, and M. Fiebig, Reversible optical switching of antiferromagnetism in $\mathrm{TbMnO}_{3}$, Nat. Photon. 10, 653 
(2016).

[20] R. Chimata, L. Isaeva, K. Kdas, A. Bergman, B. Sanyal, J. H. Mentink, M. I. Katsnelson, T. Rasing, A. Kirilyuk, A. Kimel, O. Eriksson, and M. Pereiro, All-thermal switching of amorphous Gd-Fe alloys: Analysis of structural properties and magnetization dynamics, Phys. Rev. B 92, 094411 (2015).

[21] G. P. Zhang, Y. H. Bai, and T. F. George, A new and simple model for magneto-optics uncovers an unexpected spin switching, EPL 112, 27001 (2015).

[22] G. P. Zhang, Y. H. Bai, and T. F. George, Switching ferromagnetic spins by an ultrafast laser pulse: Emergence of giant optical spin-orbit torque, EPL 115, 57003 (2016).

[23] G. P. Zhang, Y. H. Bai, and T. F. George, Is perpendicular magnetic anisotropy essential to all-optical ultrafast spin reversal in ferromagnets? J. Phys.: Condensed Mat. 29, 425801 (2017).

[24] G. P. Zhang and W. Hübner, Laser-induced ultrafast demagnetization in ferromagnetic metal, Phys. Rev. Lett. 85, 3025 (2000).

[25] G. P. Zhang, M. S. Si, M. Murakami, Y. H. Bai, and T. F. George, Generating high-order optical and spin harmonics from ferromagnetic monolayers, Nat. Commun. 9, 3031 (2018).

[26] M. S. El Hadri, P. Pirro, C.-H. Lambert, N. Bergeard, S. Petit-Watelot, M. Hehn, G. Malinowski, F. Montaigne, Y. Quessab, R. Medapalli, E. E. Fullerton, and S. Mangin, Electrical characterization of all-optical helicity-dependent switching in ferromagnetic Hall crosses, Appl. Phys. Lett. 108, 092405 (2016).

[27] G. P. Zhang and T. F. George, Total angular momentum conservation in laser-induced femtosecond magnetism, Phys. Rev. B 78, 052407 (2008).

[28] C. Boeglin, E. Beaurepaire, V. Halte, V. Lopez-Flores, C. Stamm, N. Pontius, H. A. Dürr, and J.-Y. Bigot, Distinguishing the ultrafast dynamics of spin and orbital moments in solids, Nature 465, 458 (2010).

[29] W. Töws and G. M. Pastor, Many-body theory of ultrafast demagnetization and angular momentu transfer in ferromagnetic transition metals, Phys. Rev. Lett. 115, 217204 (2015). 


\section{Appendix A: Orbital angular momentum}

At the center of all-optical spin switching is the orbital angular momentum. Firstprinciples calculations in solids often work in the crystal momentum space. Due to the translational symmetry in solids, the orbital angular momentum is largely quenched. When a laser pulse excites the system, such a calculation surely underestimates the orbital momentum change. To see this clearly, consider a single atom subject to a laser pulse. The atom must exchange its orbital angular momentum $L$ with the light field by $\pm 1 \hbar$, i.e. the selection rule $\Delta l= \pm 1$, where $l$ is the orbital angular momentum quantum number. However, for solids, the common wisdom is that the orbital angular momentum $\left\langle L_{z}\right\rangle$ is largely quenched [27] because for every positive $m_{l}$ there is a negative $m_{l}$. Then the summation over $l$ is

$$
\sum_{l}\left\langle L_{z}\right\rangle=0
$$

Thus, a solid does not allow a big change in $L$.

To reconcile the difference between the optical selection rule and the degeneracy due to translational symmetry, it is often assumed that the increase in orbital momentum has to be quenched immediately [28, 29]. Fortunately, this paradoxical contradiction does not arise for three reasons. First, only a small portion of a sample is exposed to laser excitation, so the translational symmetry is broken and the crystal momentum is no longer a good quantum number, which favors a real space view. Second, under laser excitation, original degenerate states, which lead to the orbital momentum quenching, are no longer degenerate. The summation over degenerate states in Eq. (A1) is no longer necessary. Third, optical excitation does not only affect the longitudinal component of the orbital angular momentum $L_{z}$, but also the transverse ones $L_{x}$ and $L_{y}$. To reverse spins, transverse components are more important because they constitute the spin-orbit coupling that reverses spins. Neither $L_{x}$ nor $L_{y}$ is probed by x-ray magnetic circular dichroism (XMCD), so prior time-resolved XMCD results can not be used as evidence for the insignificance of the orbital angular momentum. More importantly which component is longitudinal or transverse depends on the quantization axis. The reason why our model is successful is mainly because the initial orbital angular momentum is treated properly. We work in the real space, so the geometry of laser excitation is taken into account realistically. 

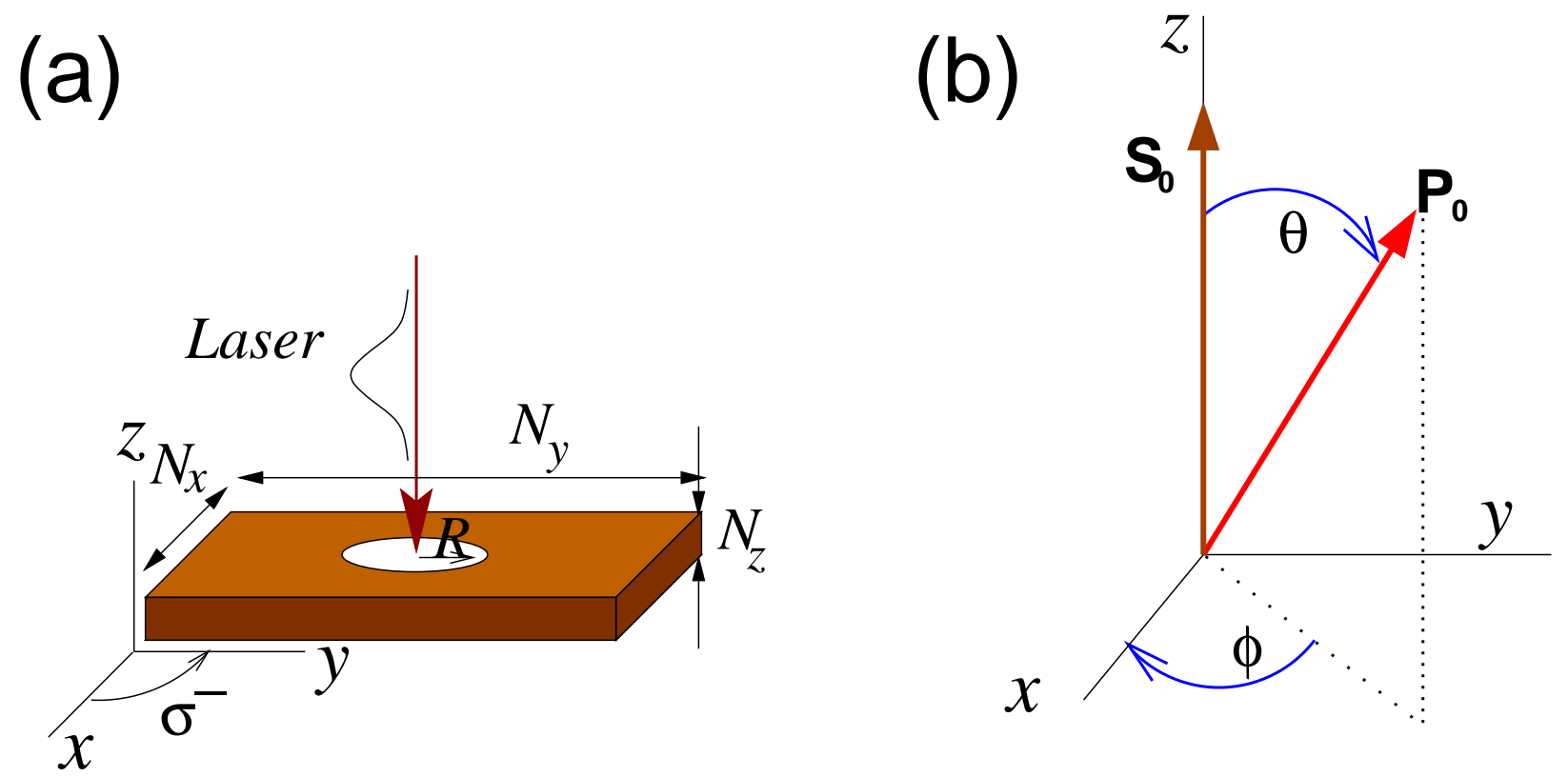

FIG. 1. (a) Schematic of our simulated magnetic thin film, with dimension $N_{x} \times N_{y} \times N_{z}$, where $N_{x}=N_{y}=501$ and $N_{z}=4$. A laser pulse impinges on the sample with radius $R$, and its polarization can be linear or circular. In the bottom of the figure, we show one example of leftcircularly polarized light $\left(\sigma^{-}\right)$. In our convention, we use the right hand rule with the thumb along the light propagation direction, and if the electric field follows the direction of the fingers, the light is right circularly polarized, otherwise, it is left-circularly polarized light. (b) Definition of the spatial orientation $(\theta, \phi)$ of the initial momentum $\mathbf{P}_{0}$ with respect to the initial spin $\mathbf{S}_{0}$ for the single spin calculation. In many-spin calculations, $\mathbf{S}_{0}$ can lie inplane or have any particular orientation. 


\section{Time (fs)}

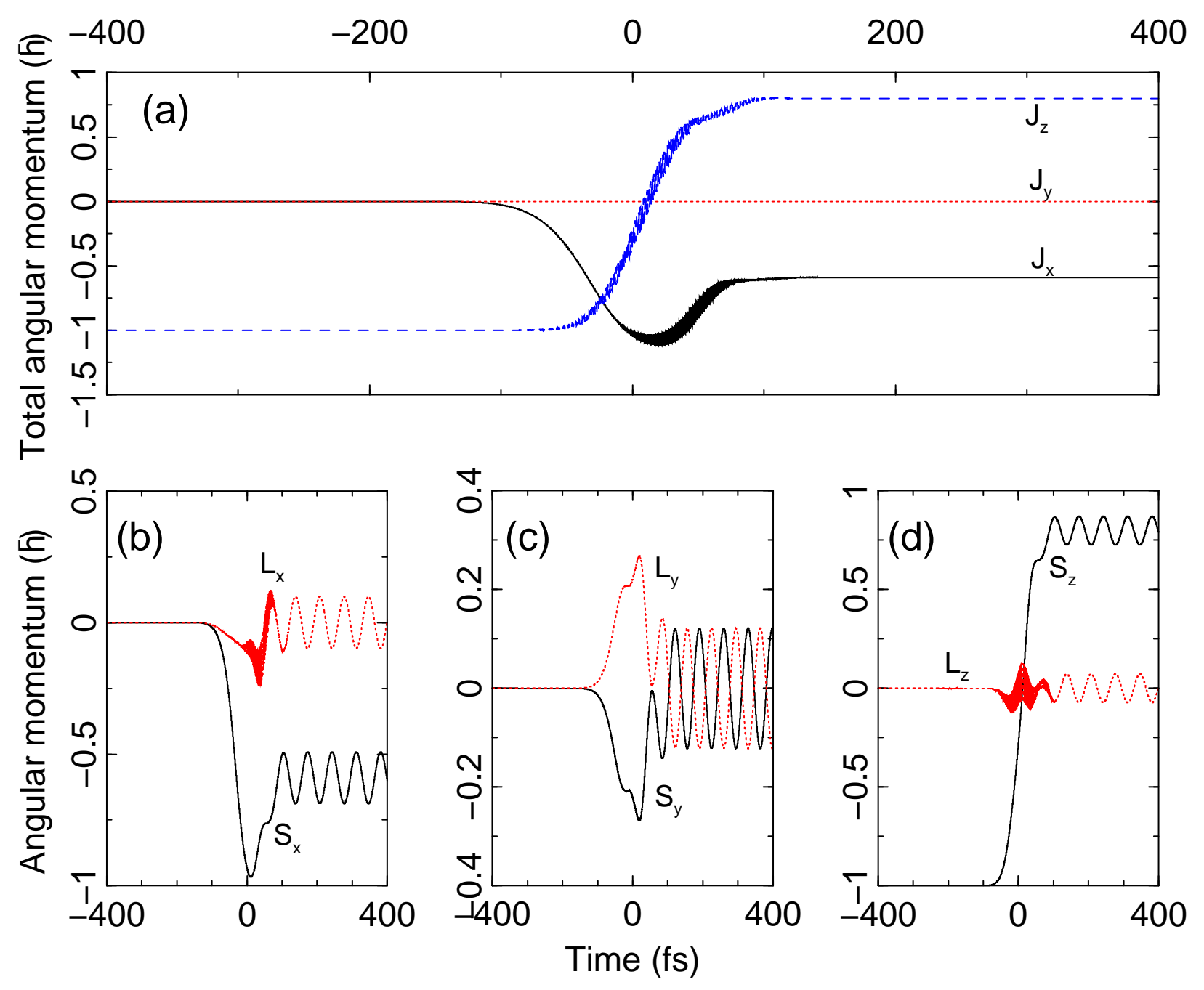

FIG. 2. (a) Total angular momentum $\mathbf{J}$ in a single spin as a function of time. Upon laser excitation, it changes rapidly. Before and after laser excitation, it is conserved. (b) The spin and orbital angular momentum along the $x$ axis. They are decoupled once the system is excited. (c) and (d) Same as (b) but for the $y$ and $z$ axes, respectively. Note that $L_{z}$ and $S_{z}$ are very different. 


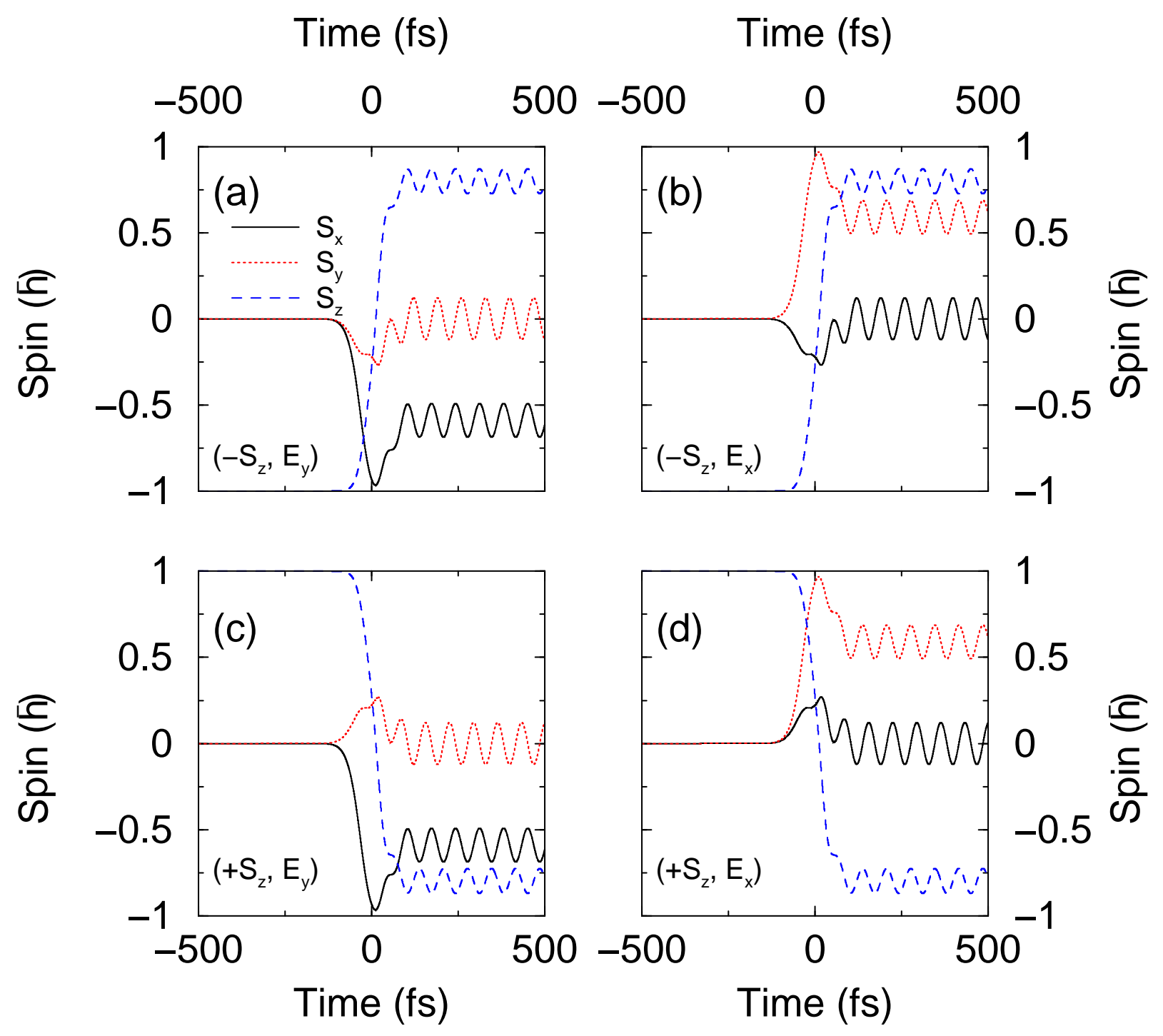

FIG. 3. Spin reversal under different electric field and spin configurations. (a) Initial spin is along the $-z$ axis and the laser electric field $\mathbf{E}(t)$ is along the $y$ axis. Spin components, $S_{x}, S_{y}$ and $S_{z}$, are denoted by the solid, dotted and dashed lines, respectively. The laser field amplitude is $0.09 \mathrm{~V} / \AA$ and the duration $T$ is 60 fs. Spin $S_{0}=1 \hbar$. (b) Initial spin is along the $-z$ axis but the electric field $\mathbf{E}(t)$ is along the $x$ axis. (c) Initial spin is along the $+z$ axis and the electric field $\mathbf{E}(t)$ is along the $y$ axis. (d) Initial spin is along the $+z$ axis and the laser electric field $\mathbf{E}(t)$ is along the $x$ axis. 

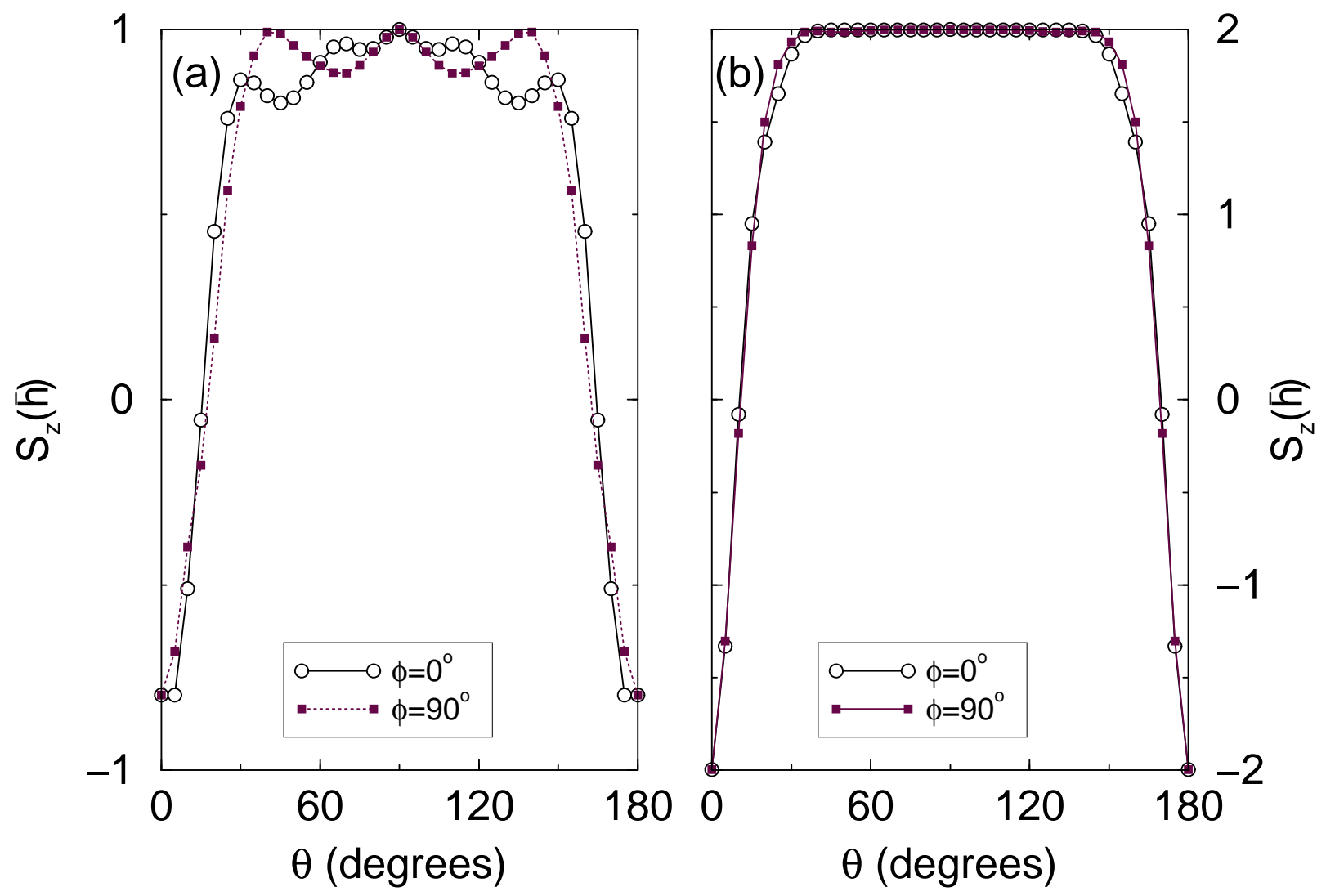

FIG. 4. (a) Dependence of the final average spin on the polar angle $\theta$ of momentum $\mathbf{P}_{0}$ at two azimuthal angles $\phi=0^{\circ}$ and $90^{\circ}$ for $S_{0}=1 \hbar$. See Fig. 1(b) as how $\theta$ and $\phi$ are defined with respect to the spin. The laser field amplitude is optimized at $A_{0}=0.09 \mathrm{~V} / \AA$, while the laser duration $T=60$ fs and photon energy $\hbar \omega=1.6 \mathrm{eV}$. The spin reversal occurs only when $\mathbf{P}_{0}$ falls into a narrow angle around $+S_{z}\left(\theta=0^{\circ}\right)$ or $-S_{z}\left(\theta=180^{\circ}\right)$. (b) Dependence of final spin on the momentum $\mathbf{P}$ orientation for $S_{0}=2 \hbar$. The laser field amplitude is optimized at $A_{0}=0.20 \mathrm{~V} / \AA$. The rest of laser parameters are the same as (a). For a larger spin, the dependence is smoother because of a larger spin-orbit torque. 

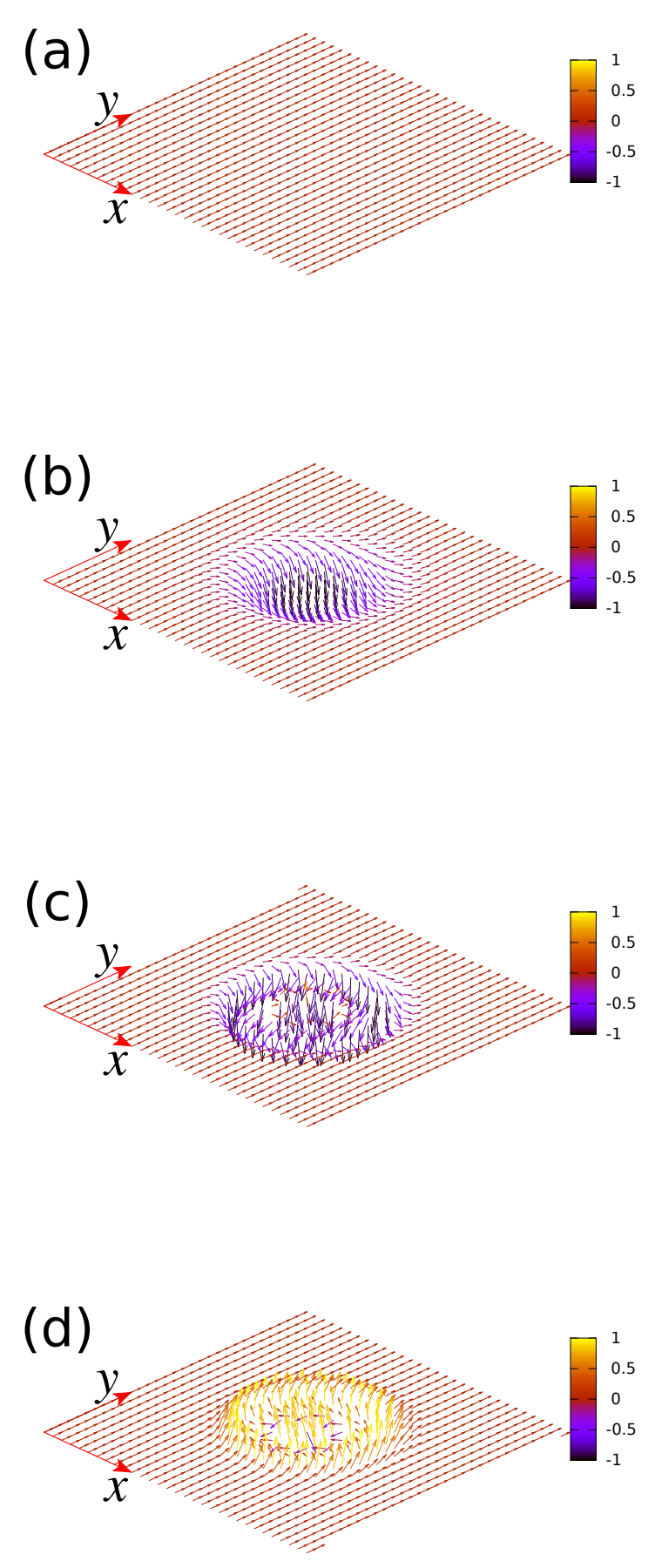

FIG. 5. Spin reversal in a uniform magnetized slab. (a) Initial spin configuration. All the spins are inplane and along the $y$ axis. There are 501 spins along both the $x$ and $y$ axes. To reduce the huge data, we only show one spin every ten spins. (b), (c) and (d) Snapshot of spins at 123 fs under linearly polarized light, right- and left-circularly polarized light, respectively. Here Figs. (b)-(d) are revised. 

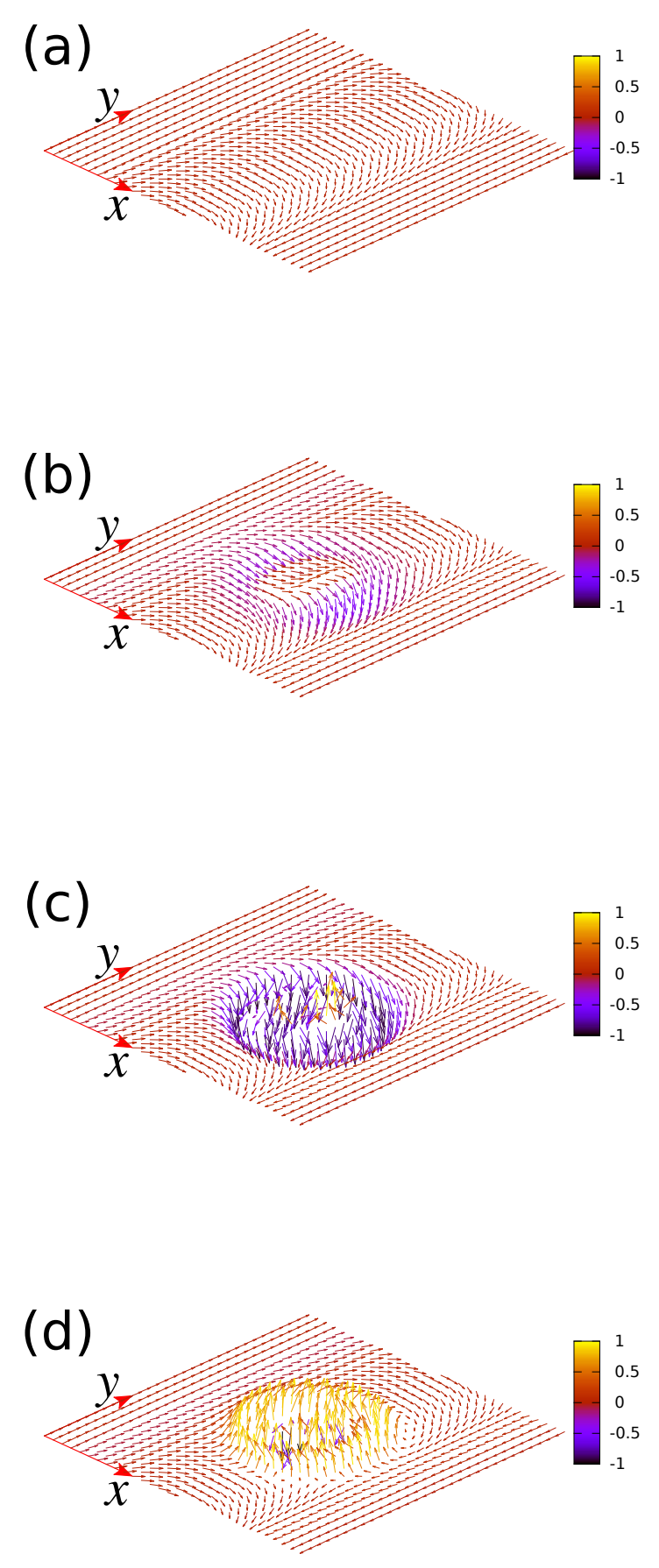

FIG. 6. Spin reversal across a Néel wall along the $y$ axis. (a) Initial spin configuration. (b), (c) and (d) Snapshot of spins at 123 fs under linearly polarized light, right- and left-circularly polarized light, respectively. $\sigma^{+}$tends to switch spin down, while $\sigma^{-}$tends to switch spin up, which creates a basin (c) and mound (d) of spins, respectively. Around the vicinity of the basin and mound, spins are clearly reversed. 

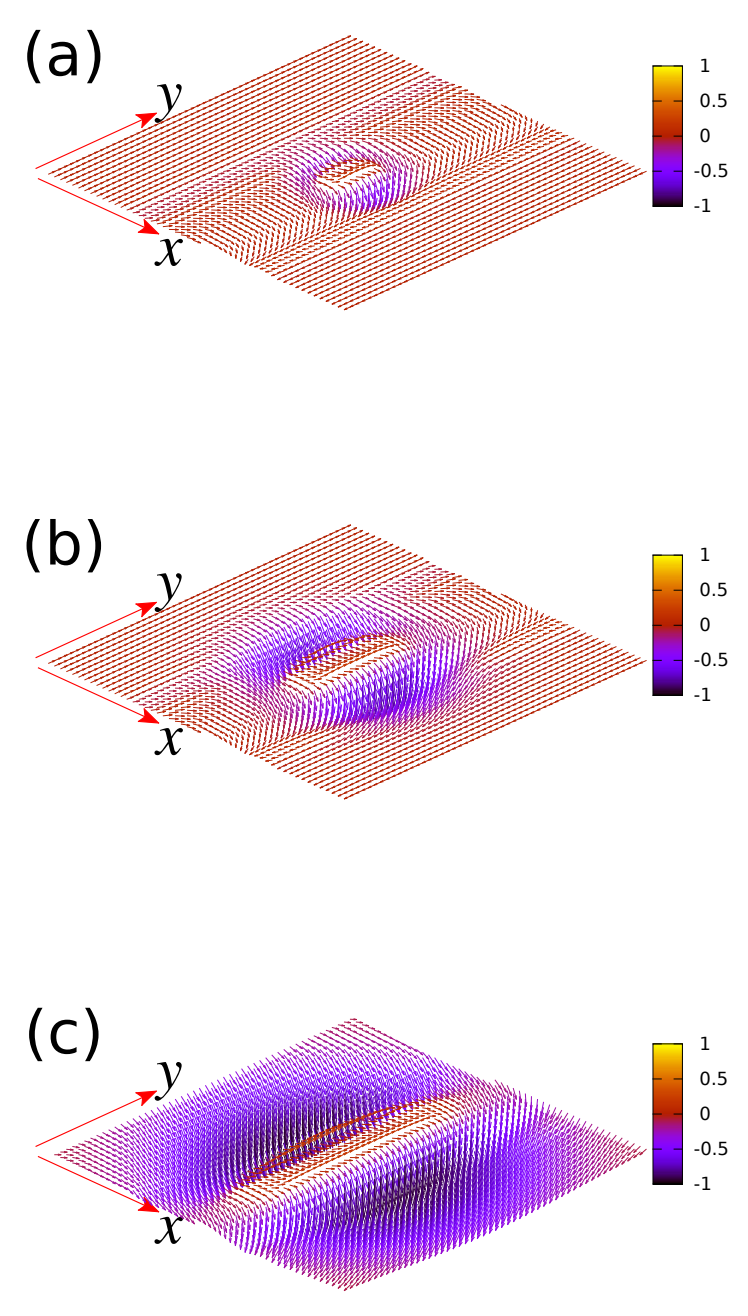

FIG. 7. Effect of laser beam radius on the spin switching in Néel walls under linearly polarized light along the $x$ axis. (a) $R=50$. (b) $R=150$. (c) $R=200$. It is clear that as $R$ increases, the affected region becomes larger. Interestingly, the original wall is still visible. Note that the image is on a much larger scale than that in Fig. 6, 

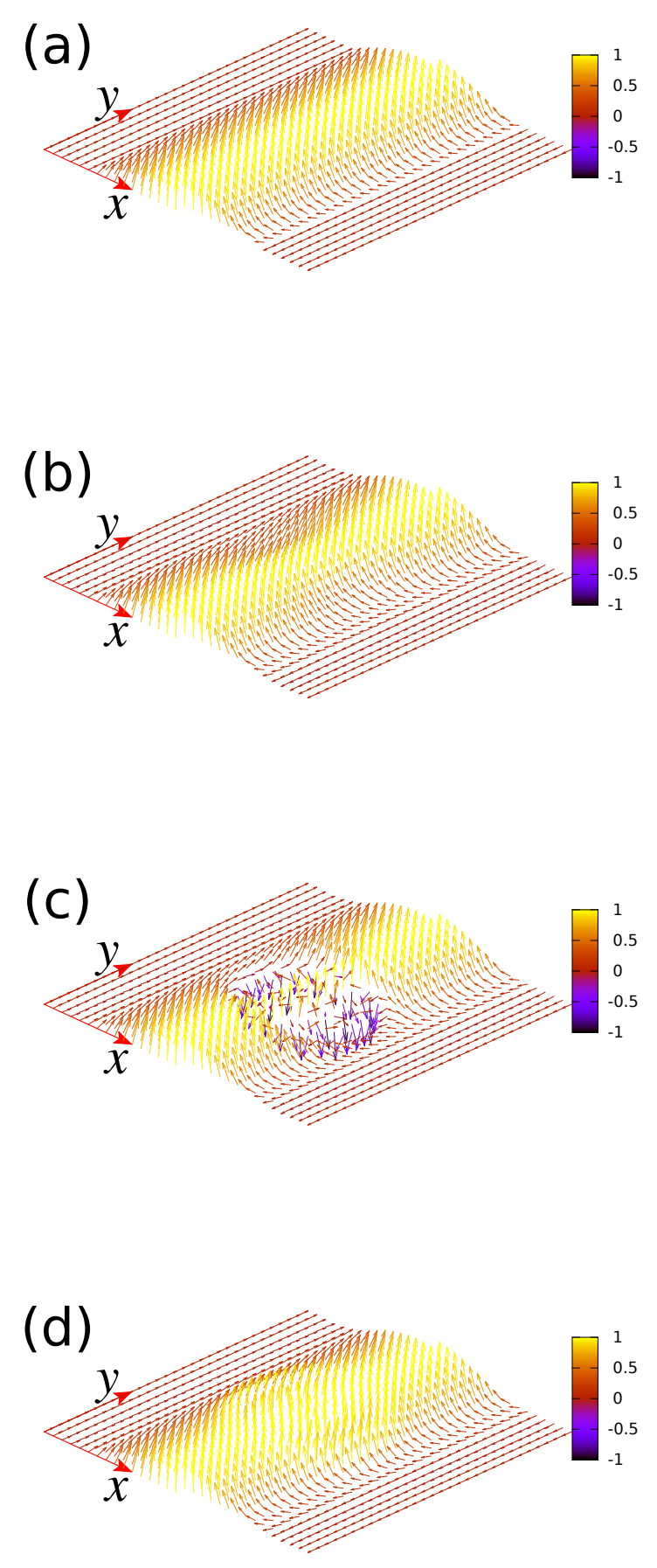

FIG. 8. Spin reversal across a Bloch wall along the $y$ axis. (a) Initial spin configuration. (b), (c) and (d) Snapshot of spins at 123 fs under linearly polarized light, right- and left-circularly polarized light, respectively. A dramatic impact on the wall is from the $\sigma^{+}$light, where the domain wall is disrupted. Changes due to the $\sigma^{-}$light are small due to its helicity [16]. 

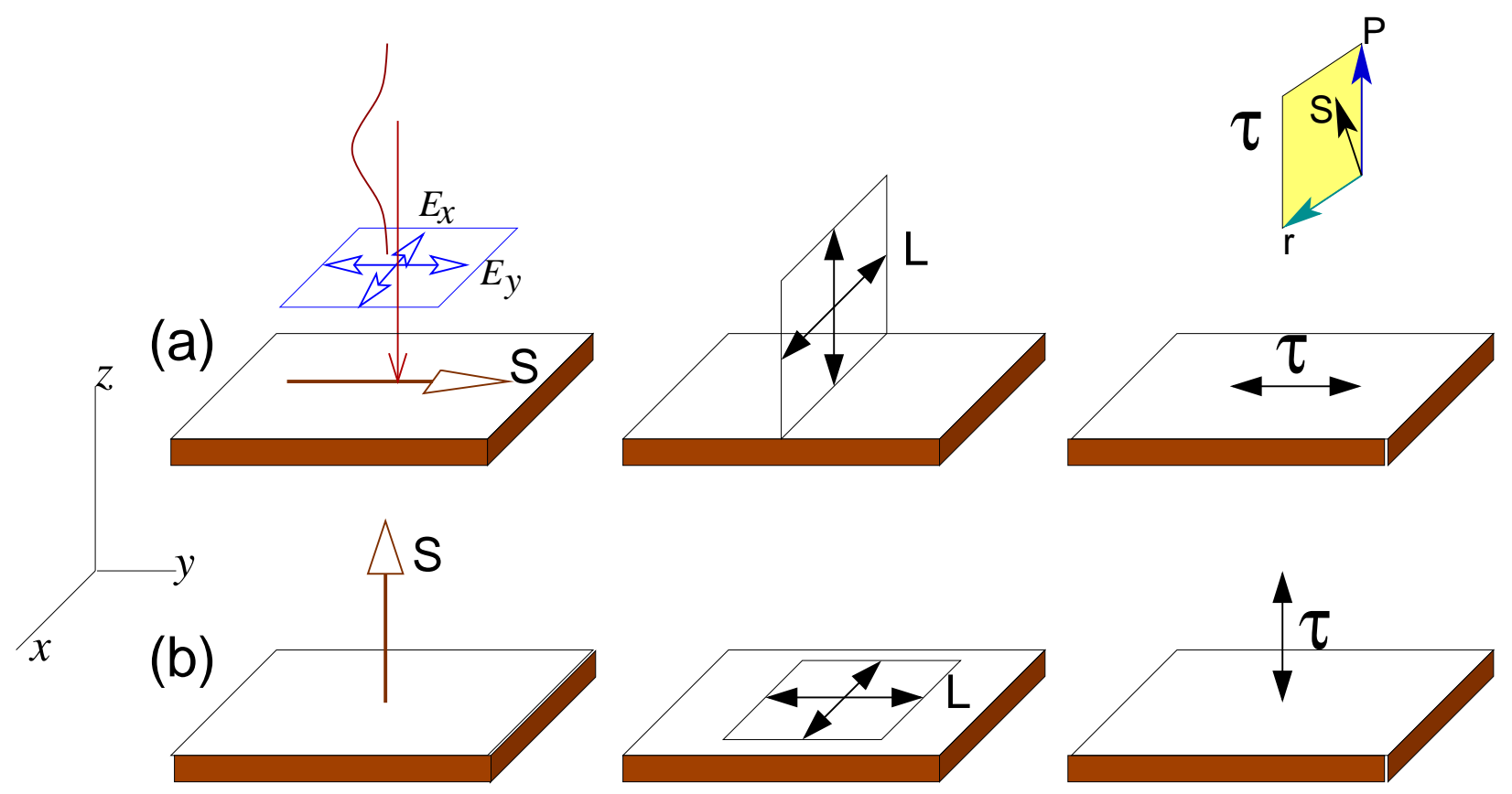

FIG. 9. Basic physics of all-optical spin switching. Here a laser pulse impinges a sample at normal incidence along the $-z$ axis, so the electric field falls in the $x y$ plane. (a) Spin is in plane along the $y$ axis. Middle: Plane of orbital angular momentum $\mathbf{L}$; Right: Spin-orbit torque is along the $y$ axis. (Top right): Relation among $\mathbf{S}, \mathbf{r}, \mathbf{P}$, and $\tau$. (b) Spin is out of plane along the $z$ axis. Middle: Plane of orbital angular momentum $\mathbf{L}$ is in the $x y$ plane; Right: Spin-orbit torque is along the $z$ axis. 